\title{
Stability and Hopf bifurcation analysis in a fractional-order delayed paddy ecosystem
}

\author{
Xiaoli Zhou' ${ }^{1}$ Zhaohua Wu' ${ }^{1}$, Zhiming Wang ${ }^{1}$ and Tiejun Zhou ${ }^{1,2^{*}}$ (D)
}

"Correspondence:

hntjzhou@126.com

${ }^{1}$ College of Science, Hunan

Agricultural University, Changsha, China

${ }^{2}$ College of Orient Science and

Technology, Hunan Agricultural

University, Changsha, China

\begin{abstract}
By introducing a delayed fractional-order differential equation model, we deal with the dynamics of the stability and Hopf bifurcation of a paddy ecosystem with three main components: rice, weeds, and inorganic fertilizer. In the system, there exists an equilibrium for rice and weeds extinction and an equilibrium for rice extinction or weeds extinction. We obtain sufficient conditions for the stability and Hopf bifurcation by analyzing their characteristic equation. Some numerical simulations validate our theoretical results.
\end{abstract}

Keywords: Paddy ecosystem; Fractional order; Delay; Stability; Hopf bifurcation

\section{Introduction}

Rice is one of the major grain crops in the world. China is the largest rice producer and consumer country in the world, where over $60 \%$ of the population is staple food for rice. Throughout the world rice producing countries, it is a major research topic to improve rice yield and quality. Obviously, there are a lot of factors affecting the production of rice, such as weed, insect, microorganism, inorganic fertilizer, light intensity, moisture, and so on. These factors interact and transform each other to form a complex nonlinear relationship. It is a common research method to analyze the interaction of all factors in a population system by using mathematical models [1-11]. As far as we know, there are only a few mathematical models that have been established for paddy ecosystems [12-14].

A differential equation model of a paddy ecosystem in fallow season was proposed by Xiang et al. [14]. They revealed the interaction between weeds and inorganic fertilizer and found that in the system, there exists a stable node, an unstable saddle point, or a saddlenode point. By considering the effects of herbivores on the paddy ecosystem in fallow season, Xiang, Wu, and Zhou found that the content of inorganic fertilizer is improved by putting some herbivores into the paddy ecosystem in fallow season. They also found that the system can exhibit Hopf bifurcation phenomenon and gave the critical value of Hopf bifurcation by taking a system parameter as the bifurcation parameter [13]. Wang et al. [12] further studied the interaction of rice, weeds, and inorganic fertilizer in a paddy ecosystem. They discussed the existence and stability of equilibria in a paddy ecosystem. They also found that there exist Hopf bifurcations in such a system.

(c) The Author(s) 2018. This article is distributed under the terms of the Creative Commons Attribution 4.0 International License (http://creativecommons.org/licenses/by/4.0/), which permits unrestricted use, distribution, and reproduction in any medium, provided you give appropriate credit to the original author(s) and the source, provide a link to the Creative Commons license, and indicate if changes were made. 
The three models mentioned have been restricted to integer-order (delay) differential equations [12-14]. In recent more than 20 years, the research of fractional-order differential equations has been the concern of many scholars. According to the study and numerical experiments in different fields such as physical, mechanical, and engineering problems, many phenomena can be described more successfully by using factional-order differential equation models. In view of this, some scholars have used fractional differential equations to study the interaction relationship of biological populations [15-20]. Recently, some researchers have also concerned about the existence of Hopf bifurcation of fractional-order models [21-27]. Abdelouahab et al. [21] obtained the Hopf bifurcation conditions of a three-dimensional fractional-order system without time delay, and Li et al. [23] obtained the Hopf bifurcation conditions of a four-dimensional fractional-order system without time delay. For general delayed fractional-order systems, the Hopf bifurcation conditions were proposed by Xiao et al. [26] in 2017.

It is of practical significance to study whether there exists a Hopf bifurcation in a paddy ecosystem. If a Hopf bifurcation exists in a paddy ecosystem, the stability of the system will be destroyed. An unstable paddy ecosystem brings difficulties and uncertainties to management of rice production. Therefore, we want to delay or eliminate the Hopf bifurcation by using the existence conditions of Hopf bifurcation. On the other hand, in case the paddy ecosystem has come up with a Hopf bifurcation, we should try to harvest rice at the peak of its biomass to increase rice yield.

In this paper, we establish a factional-order differential equation model with delay for the interaction among the main components of a paddy ecosystem. We give a detailed stability analysis of the system equilibria and study the existence of Hopf bifurcation by using the Hopf bifurcation conditions proposed by Xiao et al. [26].

\section{Preliminaries}

Considering a general delayed fractional-order system

$$
D^{\alpha} Y(t)=F(Y(t), Y(t-\tau))
$$

the time delay $\tau>0$, where $Y(t)=\left(y_{1}(t), y_{2}(t), \ldots, y_{n}(t)\right)^{T} \in \mathbb{R}^{n}$, and $D^{\alpha}$ is the Caputo fractional derivative defined as

$$
D^{\alpha} f(t)=\frac{1}{\Gamma(m-\alpha)} \int_{0}^{t}(t-s)^{m-\alpha-1} f^{(m)}(s) d s,
$$

where $\Gamma(q)=\int_{0}^{\infty} e^{-t} t^{q-1} d t$ is the gamma function, $m \in \mathbb{N}$, and $m-1<\alpha<m$. When $\alpha=m$, $D^{\alpha} f(t)=f^{(m)}(t)$. In this paper, we suppose $0<\alpha \leq 1$.

The equilibrium $Y^{*}$ of system (1) is the solution to equation $F(Y, Y)=0$.

The corresponding linearized system of (1) at an equilibrium $Y^{*}$ is of the form

$$
D^{\alpha} X(t)=A X(t)+B X(t-\tau) .
$$

The characteristic equation of system (2) is

$$
\Delta(\lambda)=\operatorname{det}\left(\lambda^{\alpha} E-A-B e^{-\lambda \tau}\right)=0 .
$$


If $\tau=0$, then system (2) is simplified as

$$
D^{\alpha} X(t)=M X(t)
$$

where the coefficient matrix $M=A+B$.

Based on the characteristic equation $\Delta(\lambda)=0$ and the coefficient matrix $M$, we have the following stability result on the delayed fractional-order system (2) [28].

Lemma 1 If $\alpha \in(0,1)$, then all the eigenvalues $\lambda$ of $M$ satisfy $|\arg (\lambda)|>\pi / 2$, and the characteristic equation $\Delta(\lambda)=0$ has no purely imaginary roots for any $\tau>0$, then the zero solution of system (2) is Lyapunov globally asymptotically stable.

The Hopf bifurcation conditions were proposed in [26] for the general delayed fractional-order system (1). If the following conditions hold, then system (1) undergoes a Hopf bifurcation at the equilibrium $Y^{*}$ when $\tau=\tau_{0}$.

(1) All the eigenvalues of the coefficient matrix $M$ of the linearized system of (1) satisfy $|\arg (\lambda)|>\alpha \pi / 2$.

(2) The characteristic equation $\Delta(\lambda)=0$ of the linearized system of (1) has a pair of purely imaginary roots $\pm i \omega_{0}$ when $\tau=\tau_{0}$.

(3) $\left.\frac{d \operatorname{Re}(\lambda(\tau))}{d \tau}\right|_{\tau=\tau_{0}}>0$, where $\operatorname{Re}(\cdot)$ denotes the real part of a complex number.

\section{Fractional-order model of a paddy ecosystem}

Wang et al. [12] have considered the following paddy ecosystem with three main components, rice, weeds, and inorganic fertilizer:

$$
\left\{\begin{array}{l}
\dot{r}(t)=c_{1} s_{1} u(t) r(t)-d_{1} r(t) \\
\dot{p}(t)=c_{2} s_{2} u(t) p(t)-d_{2} p(t) \\
\dot{u}(t)=b+d_{1} r(t-\tau)+d_{2} p(t-\tau)-s_{1} u(t) r(t)-s_{2} u(t) p(t)-d_{3} u(t),
\end{array}\right.
$$

where $r(t)$ and $p(t)$ denote the rice and weeds biomasses per unit area at time $t$, respectively, and $u(t)$ denotes the inorganic fertilizer content per unit area at time $t$. The system can reflect the interactions among rice, weeds, and inorganic fertilizer. The first two equations in system (4) indicate that the growth of rice $r(t)$ and weeds $p(t)$ are affected by soil fertility $u(t)$, light and other factors $s_{i}$, and there is natural death $d_{1} r(t)$ and $d_{2} p(t)$ for the rice and weeds. The coefficients $c_{1}$ and $c_{2}$ represent rice and weeds utilization rate of inorganic fertilizer, light energy, and other factors, respectively. The third equation in system (4) shows that the inorganic fertilizer in soil partly comes from fertilization $b$ and partly comes from organic fertilizer such as decaying leaves of rice and weeds, $d_{1} r(t-\tau)$ and $d_{2} p(t-\tau)$, which can be transformed to inorganic fertilizer after some time $\tau$ by microbial. Natural loss $d_{3} u(t)$ also reduces the content of inorganic fertilizers in soil.

Using the Caputo fractional-order derivative of order $\alpha \in(0,1)$, a fractional-order delayed paddy ecosystem is established as follows:

$$
\left\{\begin{aligned}
D^{\alpha} r(t)= & c_{1} s_{1} u(t) r(t)-d_{1} r(t), \\
D^{\alpha} p(t)= & c_{2} s_{2} u(t) p(t)-d_{2} p(t), \\
D^{\alpha} u(t)= & b+c_{3} d_{1} r(t-\tau)+c_{4} d_{2} p(t-\tau)-s_{1} u(t) r(t) \\
& -s_{2} u(t) p(t)-d_{3} u(t),
\end{aligned}\right.
$$


where $c_{3}$ and $c_{4}$ are the conversion rates from organic fertilizer $d_{1} r(t-\tau)$ and $d_{2} p(t-\tau)$ to inorganic fertilizer $u(t)$, respectively. The meaning of other symbols in system (5) are consistent with system (4). Similarly, the parameters in system (5) are nonnegative and satisfy the following conditions: $0<c_{i}<1, b \geq 0, \tau \geq 0, s_{i}>0$, and $d_{i}>0$. We also introduce the following notation [12]:

$$
\theta_{1}=\frac{d_{1}}{c_{1} s_{1}}, \quad \theta_{2}=\frac{d_{2}}{c_{2} s_{2}}
$$

where $\theta_{1}$ is called the relative mortality of rice, and $\theta_{2}$ is called the relative mortality of weeds.

\section{The stability of equilibria and Hopf bifurcation}

Similarly to [12], system (5) always has an equilibrium for rice and weeds extinction

$$
\left(r_{1}^{*}, p_{1}^{*}, u_{1}^{*}\right)=\left(0,0, \frac{b}{d_{3}}\right)
$$

If $b / d_{3}>\theta_{1}$, then system (5) has an equilibrium for weeds extinction

$$
\left(r_{2}^{*}, p_{2}^{*}, u_{2}^{*}\right)=\left(\frac{b-d_{3} \theta_{1}}{s_{1} \theta_{1}\left(1-c_{1} c_{3}\right)}, 0, \theta_{1}\right)
$$

If $b / d_{3}>\theta_{2}$, then system (5) still has an equilibrium for rice extinction

$$
\left(r_{3}^{*}, p_{3}^{*}, u_{3}^{*}\right)=\left(0, \frac{b-d_{3} \theta_{2}}{s_{2} \theta_{2}\left(1-c_{2} c_{4}\right)}, \theta_{2}\right)
$$

For an equilibrium $\left(r^{*}, p^{*}, u^{*}\right)$ of system (5), we make a coordinate transformation $x=$ $r-r^{*}, y=p-p^{*}, z=u-u^{*}$; then system (5) can be converted to

$$
\left\{\begin{aligned}
D^{\alpha} x(t)= & c_{1} s_{1}\left(u^{*}-\theta_{1}\right) x(t)+c_{1} s_{1} r^{*} z(t)+c_{1} s_{1} x(t) z(t) \\
D^{\alpha} y(t)= & c_{2} s_{2}\left(u^{*}-\theta_{2}\right) y(t)+c_{2} s_{2} p^{*} z(t)+c_{2} s_{2} y(t) z(t) \\
D^{\alpha} z(t)= & c_{3} d_{1} x(t-\tau)-s_{1} u^{*} x(t)+c_{4} d_{2} y(t-\tau)-s_{2} u^{*} y(t) \\
& -\left(s_{1} r^{*}+s_{2} p^{*}+d_{3}\right) z(t)-s_{1} x(t) z(t)-s_{2} y(t) z(t) .
\end{aligned}\right.
$$

Obviously, the linearized system of (6) is

$$
\left\{\begin{aligned}
D^{\alpha} x(t)= & c_{1} s_{1}\left(u^{*}-\theta_{1}\right) x(t)+c_{1} s_{1} r^{*} z(t) \\
D^{\alpha} y(t)= & c_{2} s_{2}\left(u^{*}-\theta_{2}\right) y(t)+c_{2} s_{2} p^{*} z(t) \\
D^{\alpha} z(t)= & c_{3} d_{1} x(t-\tau)-s_{1} u^{*} x(t)+c_{4} d_{2} y(t-\tau) \\
& -s_{2} u^{*} y(t)-\left(s_{1} r^{*}+s_{2} p^{*}+d_{3}\right) z(t)
\end{aligned}\right.
$$

Its characteristic equation is

$$
\Delta(\lambda)=\left|\begin{array}{ccc}
\lambda^{\alpha}-c_{1} s_{1}\left(u^{*}-\theta_{1}\right) & 0 & -c_{1} s_{1} r^{*} \\
0 & \lambda^{\alpha}-c_{2} s_{2}\left(u^{*}-\theta_{2}\right) & -c_{2} s_{2} p^{*} \\
s_{1} u^{*}-c_{3} d_{1} e^{-\lambda \tau} & s_{2} u^{*}-c_{4} d_{2} e^{-\lambda \tau} & \lambda^{\alpha}+s_{1} r^{*}+s_{2} p^{*}+d_{3}
\end{array}\right|=0 .
$$


When the time delay $\tau=0$, the coefficient matrix of system (7) is

$$
M=\left(\begin{array}{ccc}
c_{1} s_{1}\left(u^{*}-\theta_{1}\right) & 0 & c_{1} s_{1} r^{*} \\
0 & c_{2} s_{2}\left(u^{*}-\theta_{2}\right) & c_{2} s_{2} p^{*} \\
c_{3} d_{1}-s_{1} u^{*} & c_{4} d_{2}-s_{2} u^{*} & -s_{1} r^{*}-s_{2} p^{*}-d_{3}
\end{array}\right)
$$

Next, we consider the stability of the three equilibria of system (5).

Case (I) for the equilibrium $\left(r_{1}^{*}, p_{1}^{*}, u_{1}^{*}\right)$. At this case, we have the following conclusion of the stability of the equilibrium.

Theorem 1 If $b / d_{3}<\min \left\{\theta_{1}, \theta_{2}\right\}$, then the equilibrium for rice and weeds extinction of system (5) $\left(r_{1}^{*}, p_{1}^{*}, u_{1}^{*}\right)$ is locally asymptotically stable. Otherwise, if $b / d_{3}>\min \left\{\theta_{1}, \theta_{2}\right\}$, then the equilibrium $\left(r_{1}^{*}, p_{1}^{*}, u_{1}^{*}\right)$ is unstable.

Proof At the equilibrium $\left(r_{1}^{*}, p_{1}^{*}, u_{1}^{*}\right)$, by (8) the characteristic equation of the linearized system is

$$
\Delta(\lambda)=\left|\begin{array}{ccc}
\lambda^{\alpha}-c_{1} s_{1}\left(u_{1}^{*}-\theta_{1}\right) & 0 & 0 \\
0 & \lambda^{\alpha}-c_{2} s_{2}\left(u_{1}^{*}-\theta_{2}\right) & 0 \\
s_{1} u_{1}^{*}-c_{3} d_{1} e^{-\lambda \tau} & s_{2} u_{1}^{*}-c_{4} d_{2} e^{-\lambda \tau} & \lambda^{\alpha}+d_{3}
\end{array}\right|=0
$$

So the eigenvalues satisfy $\lambda_{1}^{\alpha}=-d_{3}, \lambda_{2}^{\alpha}=c_{1} s_{1}\left(b / d_{3}-\theta_{1}\right)$, and $\lambda_{3}^{\alpha}=c_{2} s_{2}\left(b / d_{3}-\theta_{2}\right)$. Therefore the characteristic equation $\Delta(\lambda)=0$ has no purely imaginary roots for any $\tau>0$.

Similarly, the eigenvalues of the coefficient matrix $M$ are $\lambda_{1}=-d_{3}<0, \lambda_{2}=$ $c_{1} s_{1}\left(b / d_{3}-\theta_{1}\right)$, and $\lambda_{3}=c_{2} s_{2}\left(b / d_{3}-\theta_{2}\right)$.

If $b / d_{3}<\min \left\{\theta_{1}, \theta_{2}\right\}$, then the eigenvalues of the matrix $M \lambda_{2}<0$ and $\lambda_{3}<0$. By Lemma 1 the equilibrium $(0,0,0)$ of system $(7)$ is Lyapunov globally asymptotically stable. Therefore, the equilibrium $\left(r_{1}^{*}, p_{1}^{*}, u_{1}^{*}\right)$ of system (5) is locally asymptotically stable.

If $b / d_{3}>\min \left\{\theta_{1}, \theta_{2}\right\}$, then at least one of the eigenvalues $\lambda_{2}$ and $\lambda_{3}$ of the matrix $M$ is positive. Therefore the equilibrium $\left(r_{1}^{*}, p_{1}^{*}, u_{1}^{*}\right)$ is unstable under this condition.

Case (II) for the equilibrium $\left(r_{2}^{*}, p_{2}^{*}, u_{2}^{*}\right)$.

To discuss the stability of the other two equilibria, we introduce the polynomial of degree 4 with real coefficients $a=\left(1, a_{1}, a_{2}, a_{3}, a_{4}\right)$

$$
f_{a}(\xi)=\xi^{4}+a_{1} \xi^{3}+a_{2} \xi^{2}+a_{3} \xi+a_{4}
$$

and the cubic polynomial equation

$$
v^{3}-a_{2} v^{2}+\left(a_{1} a_{3}-4 a_{4}\right) v+4 a_{2} a_{4}-a_{1}^{2} a_{4}-a_{3}^{2}=0
$$

If $a_{1}>0, a_{2}<0, a_{3}>0$, and $a_{4}>0$, then $4 a_{2} a_{4}-a_{1}^{2} a_{4}-a_{3}^{2}<0$. Hence equation (11) must have a positive real root, denoted by $v_{a}$. It is noted that equation (11) can also be expressed in the form

$$
\left(\frac{a_{1} v}{2}-a_{3}\right)^{2}-4\left(\frac{a_{1}^{2}}{4}-a_{2}+v\right)\left(\frac{v^{2}}{4}-a_{4}\right)=0
$$


Therefore from equation (12) it follows that the positive real root $v_{a}$ must satisfy $\frac{v_{a}^{2}}{4}-a_{4} \geq 0$. Let

$$
M_{a}=\sqrt{\frac{a_{1}^{2}}{4}-a_{2}+v_{a}}, \quad N_{a}=\sqrt{\frac{v_{a}^{2}}{4}-a_{4}}
$$

and

$$
\Delta_{a}=\frac{a_{1}^{2}}{2}-a_{2}-v_{a}-a_{1} M_{a}+4 \operatorname{sgn}\left(\frac{a_{1} v_{a}}{2}-a_{3}\right) N_{a},
$$

where $\operatorname{sgn}(\cdot)$ is the sign function.

Lemma 2 Suppose that $a_{i}>0(i=1,3,4)$. If $a_{2}<0$ and $\Delta_{a} \geq 0$, then there are only two positive real roots of the equation $f_{a}(\xi)=\xi^{4}+a_{1} \xi^{3}+a_{2} \xi^{2}+a_{3} \xi+a_{4}=0$. If $a_{2} \geq 0$, or $a_{2}<0$ and $\Delta_{a}<0$, then the equation $f_{a}(\xi)=0$ has no positive real root.

Proof The polynomial $f_{a}(\xi)$ can be decomposed into the product of two quadratic polynomials

$$
f_{1}(\xi)=\xi^{2}+\left(\frac{a_{1}}{2}-M_{a}\right) \xi+\frac{v_{a}}{2}-\operatorname{sgn}\left(\frac{a_{1} v_{a}}{2}-a_{3}\right) N_{a}
$$

and

$$
f_{2}(\xi)=\xi^{2}+\left(\frac{a_{1}}{2}+M_{a}\right) \xi+\frac{v_{a}}{2}+\operatorname{sgn}\left(\frac{a_{1} v_{a}}{2}-a_{3}\right) N_{a} .
$$

The discriminants of the polynomials $f_{1}(\xi)$ and $f_{2}(\xi)$ are $\Delta_{a}$ and

$$
\Delta_{1}=\frac{a_{1}^{2}}{2}-a_{2}-v_{a}+a_{1} M_{a}-4 \operatorname{sgn}\left(\frac{a_{1} v_{a}}{2}-a_{3}\right) N_{a},
$$

respectively. If $\Delta_{a} \geq 0$, then the equation $f_{1}(\xi)=0$ has two real roots $\xi_{a 1}$ and $\xi_{a 2}$. From

$$
\xi_{a 1}+\xi_{a 2}=-\frac{a_{1}}{2}+M_{a}>0 \quad \text { and } \quad \xi_{a 1} \xi_{a 2}=\frac{v_{a}}{2}-\operatorname{sgn}\left(\frac{a_{1} v_{a}}{2}-a_{3}\right) N_{a}>0
$$

we haven $\xi_{a 1}>0$ and $\xi_{a 2}>0$.

If $\Delta_{1} \geq 0$, then the equation $f_{2}(\xi)=0$ has two real roots $\xi_{a 3}$ and $\xi_{a 4}$. From

$$
\xi_{a 3}+\xi_{a 4}=-\frac{a_{1}}{2}-M_{a}<0 \quad \text { and } \quad \xi_{a 3} \xi_{a 4}=\frac{v_{a}}{2}+\operatorname{sgn}\left(\frac{a_{1} v_{a}}{2}-a_{3}\right) N_{a}>0
$$

we have $\xi_{a 3}<0$ and $\xi_{a 4}<0$.

Therefore equation $f_{a}(\xi)=0$ has only two positive real roots.

Otherwise, if $a_{i}>0(i=1,3,4)$ and $a_{2} \geq 0$, it is obvious that the equation $f_{a}(\xi)=0$ has no positive real root. If $a_{i}>0(i=1,3,4), a_{2}<0$, and $\Delta_{a}<0$, then the equation $f_{1}(\xi)=0$ has no real root, and so the equation $f_{a}(\xi)=0$ has no positive real root. 
Let the coefficients of polynomial (10) be as follows:

$$
\begin{aligned}
& a_{1}=2\left(s_{1} r_{2}^{*}+d_{3}\right) \cos \frac{\alpha \pi}{2}, \quad a_{2}=\left(s_{1} r_{2}^{*}+d_{3}\right)^{2}+2 s_{1} r_{2}^{*} d_{1} \cos (\alpha \pi), \\
& a_{3}=2 s_{1} r_{2}^{*} d_{1}\left(s_{1} r_{2}^{*}+d_{3}\right) \cos \frac{\alpha \pi}{2}, \quad a_{4}=s_{1}^{2} r_{2}^{* 2} d_{1}^{2}\left(1-c_{1}^{2} c_{3}^{2}\right) .
\end{aligned}
$$

Since $0<\alpha<1$, we have that $a_{1}>0, a_{3}>0$, and $a_{4}>0$.

The conclusion of the stability of the equilibrium $\left(r_{2}^{*}, p_{2}^{*}, u_{2}^{*}\right)$ is as follows.

Theorem 2 Suppose that $b / d_{3}>\theta_{1}$.

(I) If $\theta_{1}>\theta_{2}$, then the equilibrium $\left(r_{2}^{*}, p_{2}^{*}, u_{2}^{*}\right)$ is unstable.

(II) If $\theta_{1}<\theta_{2}$ and $a_{2} \geq 0$, or $\theta_{1}<\theta_{2}, a_{2}<0$, and $\Delta_{a}<0$, then the equilibrium $\left(r_{2}^{*}, p_{2}^{*}, u_{2}^{*}\right)$ is locally asymptotically stable for $\tau \geq 0$.

(III) If $\theta_{1}<\theta_{2}, a_{2}<0$, and $\Delta_{a}>0$, then there exists a positive number $\tau_{a}$ such that when $\tau \in\left[0, \tau_{a}\right)$, the equilibrium $\left(r_{2}^{*}, p_{2}^{*}, u_{2}^{*}\right)$ is locally asymptotically stable; when $\tau>\tau_{a}$, the equilibrium $\left(r_{2}^{*}, p_{2}^{*}, u_{2}^{*}\right)$ is unstable; and a Hopf bifurcation emerges at $\tau=\tau_{a}$.

Proof By (8) the characteristic equation of the linearized system is

$$
\left(\lambda^{\alpha}-c_{2} s_{2}\left(\theta_{1}-\theta_{2}\right)\right)\left(\lambda^{2 \alpha}+\left(s_{1} r_{2}^{*}+d_{3}\right) \lambda^{\alpha}+s_{1} r_{2}^{*} d_{1}\left(1-c_{1} c_{3} e^{-\lambda \tau}\right)\right)=0
$$

It has one real eigenvalue satisfying $\lambda_{1}^{\alpha}=c_{2} s_{2}\left(\theta_{1}-\theta_{2}\right)$, the real part of which cannot be zero. Its other eigenvalues are the roots of the equation

$$
\lambda^{2 \alpha}+\left(s_{1} r_{2}^{*}+d_{3}\right) \lambda^{\alpha}+s_{1} r_{2}^{*} d_{1}\left(1-c_{1} c_{3} e^{-\lambda \tau}\right)=0
$$

By (9) the characteristic equation of the coefficient matrix $M$ is

$$
\left(\lambda-c_{2} s_{2}\left(\theta_{1}-\theta_{2}\right)\right)\left(\lambda^{2}+\left(s_{1} r_{2}^{*}+d_{3}\right) \lambda+s_{1} r_{2}^{*} d_{1}\left(1-c_{1} c_{3}\right)\right)=0 .
$$

It has one real eigenvalue $\lambda_{1}=c_{2} s_{2}\left(\theta_{1}-\theta_{2}\right)$. Its other eigenvalues are

$$
\lambda_{2,3}=\frac{1}{2}\left(-\left(s_{1} r_{2}^{*}+d_{3}\right) \pm \sqrt{\left(s_{1} r_{2}^{*}+d_{3}\right)^{2}-4 s_{1} r_{2}^{*} d_{1}\left(1-c_{1} c_{3}\right)}\right) .
$$

Obviously, the real parts of $\lambda_{2,3}$ are less than zero.

(I) If $\theta_{1}>\theta_{2}$, then the eigenvalue of the coefficient matrix $M \lambda_{1}>0$. It indicates that the equilibrium $\left(r_{2}^{*}, p_{2}^{*}, u_{2}^{*}\right)$ is unstable.

(II) If $\theta_{1}<\theta_{2}$, then the real eigenvalue of the coefficient matrix $M \lambda_{1}<0$. So the eigenvalues $\lambda_{j}$ of $M$ satisfy $\left|\arg \left(\lambda_{j}\right)\right|>\frac{\pi}{2}>\frac{\alpha \pi}{2}(j=1,2,3)$.

Assume that equation (17) has a purely imaginary $\operatorname{root} \lambda=i \xi=\xi\left(\cos \frac{\pi}{2}+i \sin \frac{\pi}{2}\right)(\xi>0)$. Substituting it into (17) gives

$$
\begin{aligned}
& \xi^{2 \alpha}(\cos \alpha \pi+i \sin \alpha \pi)+\left(s_{1} r_{2}^{*}+d_{3}\right) \xi^{\alpha}\left(\cos \frac{\alpha \pi}{2}+i \sin \frac{\alpha \pi}{2}\right) \\
& +s_{1} r_{2}^{*} d_{1}\left(1-c_{1} c_{3} \cos \tau \xi+i c_{1} c_{3} \sin \tau \xi\right)=0 .
\end{aligned}
$$


Separating its real and imaginary parts yields

$$
\xi^{2 \alpha} \cos \alpha \pi+\xi^{\alpha}\left(s_{1} r_{2}^{*}+d_{3}\right) \cos \frac{\alpha \pi}{2}+s_{1} r_{2}^{*} d_{1}\left(1-c_{1} c_{3} \cos \tau \xi\right)=0
$$

and

$$
\xi^{2 \alpha} \sin \alpha \pi+\xi^{\alpha}\left(s_{1} r_{2}^{*}+d_{3}\right) \sin \frac{\alpha \pi}{2}+c_{1} c_{3} s_{1} r_{2}^{*} d_{1} \sin \tau \xi=0 .
$$

So we have

$$
\begin{aligned}
& \cos \tau \xi=\frac{\xi^{2 \alpha} \cos \alpha \pi+\xi^{\alpha}\left(s_{1} r_{2}^{*}+d_{3}\right) \cos \frac{\alpha \pi}{2}+s_{1} r_{2}^{*} d_{1}}{c_{1} c_{3} s_{1} r_{2}^{*} d_{1}}, \\
& \sin \tau \xi=-\frac{\xi^{2 \alpha} \sin \alpha \pi+\xi^{\alpha}\left(s_{1} r_{2}^{*}+d_{3}\right) \sin \frac{\alpha \pi}{2}}{c_{1} c_{3} s_{1} r_{2}^{*} d_{1}}
\end{aligned}
$$

Since $\sin ^{2} \tau \xi+\cos ^{2} \tau \xi=1$, we have

$$
\begin{aligned}
& \left(\xi^{2 \alpha} \cos \alpha \pi+\xi^{\alpha}\left(s_{1} r_{2}^{*}+d_{3}\right) \cos \frac{\alpha \pi}{2}+s_{1} r_{2}^{*} d a_{1}\right)^{2} \\
& +\left(\xi^{2 \alpha} \sin \alpha \pi+\xi^{\alpha}\left(s_{1} r_{2}^{*}+d_{3}\right) \sin \frac{\alpha \pi}{2}\right)^{2}=c_{1}^{2} c_{3}^{2} s_{1}^{2} r_{2}^{* 2} d_{1}^{2},
\end{aligned}
$$

that is,

$$
f_{a}\left(\xi^{\alpha}\right)=\xi^{4 \alpha}+a_{1} \xi^{3 \alpha}+a_{2} \xi^{2 \alpha}+a_{3} \xi^{\alpha}+a_{4}=0
$$

where $a_{i}(i=1,2,3,4)$ are given in (15) and (16); note that $a_{1}, a_{3}, a_{4}>0$. If $a_{2} \geq 0$, or $a_{2}<0$ and $\Delta_{a}<0$, then the equation $f_{a}(\xi)=0$ has no positive real root by Lemma 2 . This leads to that equation (20) has no any positive real number $\xi$. Therefore the real parts of any roots of (17) must be negative for any $\tau>0$. This shows that the equilibrium $\left(r_{2}^{*}, p_{2}^{*}, u_{2}^{*}\right)$ is locally asymptotically stable for any $\tau \geq 0$.

(III) If $\theta_{1}<\theta_{2}, a_{2}<0$, and $\Delta_{a}>0$, then the equation $f_{a}(\xi)=0$ has two unequal positive real roots by Lemma 2 ; denote the larger root by $\xi_{+}$. So equation (20) has a positive real $\operatorname{root} \xi_{a}$ satisfying $\xi_{a}^{\alpha}=\xi_{+}$.

Notice that $\sin \tau_{a} \xi_{a}<0$ from (19). If $\cos \tau_{a} \xi_{a}>0$, then from (19) we have

$$
\tau_{a}=\frac{2 \pi}{\xi_{a}}-\frac{1}{\xi_{a}} \arcsin \frac{\xi_{a}^{2 \alpha} \sin \alpha \pi+\xi_{a}^{\alpha}\left(s_{1} r_{2}^{*}+d_{3}\right) \sin \frac{\alpha \pi}{2}}{c_{1} c_{3} s_{1} r_{2}^{*} d_{1}} .
$$

If $\cos \tau_{a} \xi_{a}<0$, then we have

$$
\tau_{a}=\frac{\pi}{\xi_{a}}+\frac{1}{\xi_{a}} \arcsin \frac{\xi_{a}^{2 \alpha} \sin \alpha \pi+\xi_{a}^{\alpha}\left(s_{1} r_{2}^{*}+d_{3}\right) \sin \frac{\alpha \pi}{2}}{c_{1} c_{3} s_{1} r_{2}^{*} d_{1}} .
$$

Next, we verify the transversal condition. Taking the derivative of $\lambda$ with respect to $\tau$ in (17), we have

$$
\frac{d \lambda}{d \tau}=-\frac{c_{1} c_{3} s_{1} r_{2}^{*} d_{1} \lambda e^{-\lambda \tau}}{2 \alpha \lambda^{2 \alpha-1}+\alpha\left(s_{1} r_{2}^{*}+d_{3}\right) \lambda^{\alpha-1}+c_{1} c_{3} s_{1} r_{2}^{*} d_{1} \tau e^{-\lambda \tau}} .
$$


So we obtain

$$
\begin{aligned}
\left.\left(\frac{d \lambda}{d \tau}\right)^{-1}\right|_{\lambda=i \xi_{a}}= & -\left.\left[\frac{2 \alpha \lambda^{2 \alpha-2} e^{\lambda \tau}+\alpha\left(s_{1} r_{2}^{*}+d_{3}\right) \lambda^{\alpha-2} e^{\lambda \tau}}{c_{1} c_{3} s_{1} r_{2}^{*} d_{1}}+\frac{\tau}{\lambda}\right]\right|_{\lambda=i \xi \xi_{a}} \\
= & -\frac{\alpha \xi_{a}^{\alpha-2}}{c_{1} c_{3} s_{1} r_{2}^{*} d_{1}}\left[2 \xi_{a}^{\alpha}\left(\cos \frac{\pi}{2}+i \sin \frac{\pi}{2}\right)^{2 \alpha-2} e^{i \xi_{a} \tau_{a}}\right. \\
& \left.+\left(s_{1} r_{2}^{*}+d_{3}\right)\left(\cos \frac{\pi}{2}+i \sin \frac{\pi}{2}\right)^{\alpha-2} e^{i \xi_{a} \tau_{a}}\right]-\frac{\tau_{a}}{i \xi_{a}}
\end{aligned}
$$

Thus, its real part is

$$
\begin{aligned}
\operatorname{Re}\left\{\left.\left(\frac{d \lambda}{d \tau}\right)^{-1}\right|_{\lambda=i \xi_{a}}\right\}= & -\frac{\alpha \xi_{a}^{\alpha-2}}{c_{1} c_{3} s_{1} r_{2}^{*} d_{1}}\left[2 \xi_{a}^{\alpha}\left(\cos (\alpha-1) \pi \cos \xi_{a} \tau_{a}-\sin (\alpha-1) \pi \sin \xi_{a} \tau_{a}\right)\right. \\
& \left.+\left(s_{1} r_{2}^{*}+d_{3}\right)\left(\cos \frac{(\alpha-2) \pi}{2} \cos \xi_{a} \tau_{a}-\sin \frac{(\alpha-2) \pi}{2} \sin \xi_{a} \tau_{a}\right)\right] \\
= & \frac{\alpha \xi_{a}^{\alpha-2}}{c_{1} c_{3} s_{1} r_{2}^{*} d_{1}}\left[\cos \xi_{a} \tau_{a}\left(2 \xi_{a}^{\alpha} \cos \alpha \pi+\left(s_{1} r_{2}^{*}+d_{3}\right) \cos \frac{\alpha \pi}{2}\right)\right. \\
& \left.-\sin \xi_{a} \tau_{a}\left(2 \xi_{a}^{\alpha} \sin \alpha \pi+\left(s_{1} r_{2}^{*}+d_{3}\right) \sin \frac{\alpha \pi}{2}\right)\right]
\end{aligned}
$$

Substituting (18) and (19) into this expression, we obtain

$$
\begin{aligned}
\operatorname{Re}\left\{\left.\left(\frac{d \lambda}{d \tau}\right)^{-1}\right|_{\lambda=i \xi_{a}}\right\}= & \frac{\alpha \xi_{a}^{\alpha-2}}{\left(c_{1} c_{3} s_{1} r_{2}^{*} d_{1}\right)^{2}}\left[2 \xi_{a}^{3 \alpha}+3\left(s_{1} r_{2}^{*}+d_{3}\right) \cos \frac{\alpha \pi}{2} \xi_{a}^{2 \alpha}\right. \\
& \left.+\left(s_{1} r_{2}^{*}+d_{3}\right)^{2} \xi_{a}^{\alpha}+s_{1} r_{2}^{*} d_{1}\left(2 \xi_{a}^{\alpha} \cos \alpha \pi+\left(s_{1} r_{2}^{*}+d_{3}\right) \cos \frac{\alpha \pi}{2}\right)\right] \\
= & \frac{\alpha \xi_{a}^{\alpha-2}}{2\left(c_{1} c_{3} s_{1} r_{2}^{*} d_{1}\right)^{2}}\left(4 \xi_{a}^{3 \alpha}+3 a_{1} \xi_{a}^{2 \alpha}+2 a_{2} \xi_{a}^{\alpha}+a_{3}\right) \\
= & \frac{\alpha \xi_{a}^{\alpha-2} f^{\prime}\left(\xi_{+}\right)}{2\left(c_{1} c_{3} s_{1} r_{2}^{*} d_{1}\right)^{2}} .
\end{aligned}
$$

Since $\xi_{+}$is the larger single root of the equation $f_{a}(\xi)=0$ and the highest order power coefficient of the polynomial is positive, we have $f^{\prime}\left(\xi_{+}\right)>0$. Therefore the transversal condition is satisfied, and thus a Hopf bifurcation occurs at $\tau=\tau_{a}$.

Remark 1 From (15) we know that if $\left(s_{1} r_{2}^{*}+d_{3}\right)^{2} \geq 2 s_{1} r_{2}^{*} d_{1}$, then $a_{2} \geq 0$. When $\left(s_{1} r_{2}^{*}+d_{3}\right)^{2}<$ $2 s_{1} r_{2}^{*} d_{1}$, we let

$$
\alpha_{a}=1-\frac{1}{\pi} \arccos \frac{\left(s_{1} r_{2}^{*}+d_{3}\right)^{2}}{2 s_{1} r_{2}^{*} d_{1}}
$$

Obviously, if $0<\alpha<\alpha_{a}$, then $a_{2}>0$. Otherwise, if $\alpha_{a}<\alpha<1$, then $a_{2}<0$.

Case (III) for the equilibrium $\left(r_{3}^{*}, p_{3}^{*}, u_{3}^{*}\right)$. 
To discuss the stability of the equilibrium $\left(r_{3}^{*}, p_{3}^{*}, u_{3}^{*}\right)$, similarly to case (II), we introduce a polynomial of degree 4 with real coefficients $q=\left(1, q_{1}, q_{2}, q_{3}, q_{4}\right)$ as follows:

$$
f_{q}(\xi)=\xi^{4}+q_{1} \xi^{3}+q_{2} \xi^{2}+q_{3} \xi+q_{4}
$$

where

$$
\begin{aligned}
& q_{1}=2\left(s_{2} p_{3}^{*}+d_{3}\right) \cos \frac{\alpha \pi}{2}, \quad q_{2}=\left(s_{2} p_{3}^{*}+d_{3}\right)^{2}+2 s_{2} p_{3}^{*} d_{2} \cos \alpha \pi, \\
& q_{3}=2 s_{2} p_{3}^{*} d_{2}\left(s_{2} p_{3}^{*}+d_{3}\right) \cos \frac{\alpha \pi}{2}, \quad q_{4}=s_{2}^{2} p_{3}^{* 2} d_{2}^{2}\left(1-c_{2}^{2} c_{4}^{2}\right) .
\end{aligned}
$$

Since $0<\alpha<1$, it is obvious that $q_{1}>0, q_{3}>0$, and $q_{4}>0$.

Similarly to case (II), we have the following stability conclusion of the equilibrium $\left(r_{3}^{*}, p_{3}^{*}, u_{3}^{*}\right)$.

Theorem 3 Suppose that $b / d_{3}>\theta_{2}$.

(I) If $\theta_{1}<\theta_{2}$, then the equilibrium $\left(r_{3}^{*}, p_{3}^{*}, u_{3}^{*}\right)$ is unstable.

(II) If $\theta_{1}>\theta_{2}$ and $q_{2} \geq 0$, or $\theta_{1}>\theta_{2}, q_{2}<0$, and $\Delta_{q}<0$, then the equilibrium $\left(r_{3}^{*}, p_{3}^{*}, u_{3}^{*}\right)$ is locally asymptotically stable for $\tau \geq 0$.

(III) If $\theta_{1}>\theta_{2}, q_{2}<0$, and $\Delta_{q}>0$, then there exists a positive number $\tau_{q}$ such that the equilibrium $\left(r_{3}^{*}, p_{3}^{*}, u_{3}^{*}\right)$ is locally asymptotically stable for $\tau \in\left[0, \tau_{q}\right)$ and unstable when $\tau>\tau_{q}$. A Hopf bifurcation emerges at the equilibrium $\left(r_{3}^{*}, p_{3}^{*}, u_{3}^{*}\right)$ when $\tau=\tau_{q}$.

The proof of Theorem 3 is similar to Theorem 2 and is omitted here.

Remark 2 From (25) we know that if $\left(s_{2} p_{3}^{*}+d_{3}\right)^{2} \geq 2 s_{2} p_{3}^{*} d_{2}$, then $q_{2} \geq 0$. When $\left(s_{2} p_{3}^{*}+d_{3}\right)^{2}<2 s_{2} p_{3}^{*} d_{2}$, we let

$$
\alpha_{q}=1-\frac{1}{\pi} \arccos \left(\frac{\left(s_{2} p_{3}^{*}+d_{3}\right)^{2}}{2 s_{2} p_{3}^{*} d_{2}}\right) .
$$

Obviously, if $0<\alpha<\alpha_{q}$, then $q_{2}>0$. Otherwise, if $\alpha_{q}<\alpha<1$, then $a_{q}<0$.

\section{Examples}

In this section, we give two examples to confirm our theoretical results obtained in Sect. 4 and use the predictor-corrector scheme to calculate their numerical solutions [29]. In system (5), we let $\alpha=0.98, c_{1}=0.8, c_{2}=0.1, c_{3}=0.9, c_{4}=0.6, s_{1}=0.6, s_{2}=0.1, d_{1}=0.9$, $d_{2}=0.1$, and $d_{3}=0.1$.

First, we take $b=1.1$. System (5) has three equilibria: the equilibrium for rice and weeds extinction $(0,0,11)$, the equilibrium for weeds extinction $(2.8968,0,1.875)$, and the equilibrium for rice extinction $(0,0.1064,10)$. By computing we have $b / d_{3}=11, \theta_{1}=1.875$, $\theta_{2}=10$, and $a_{2} \approx 0.2562>0$. So the inequality $b / d_{3}>\theta_{2}>\theta_{1}$ holds. By Theorem 2 the equilibrium $(2.8968,0,1.875)$ is asymptotically stable for any $\tau \geq 0$ as illustrated in Fig. 1 (where $\tau=13.5$ ). By Theorems 1 and 3 the equilibria $(0,0,11)$ and $(0,0.1064,10)$ are unstable.

In succession, we take $b=0.3$ again. System (5) has two equilibria: the equilibrium for rice and weeds extinction $(0,0,3)$ and the equilibrium for weeds extinction 


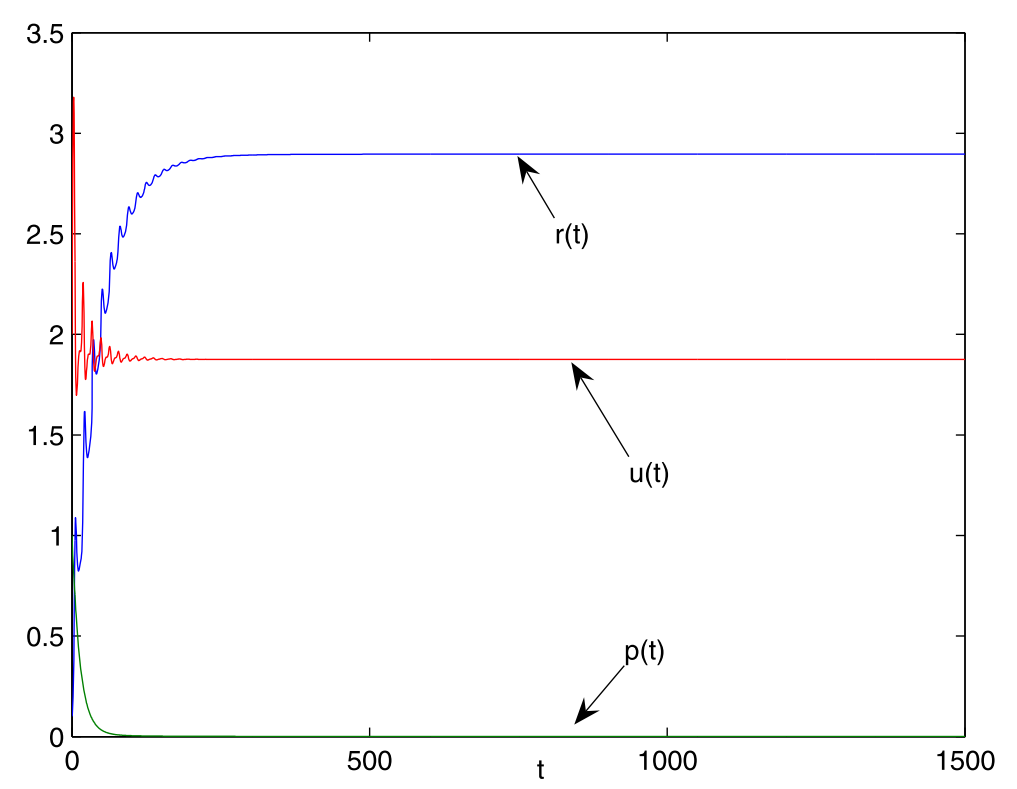

Figure 1 The asymptotical stability of $\left(r_{2}^{*}, p_{2}^{*}, u_{2}^{*}\right)=(2.8968,0,1.875)$. The parameters of system (5) are $c_{1}=0.8$, $c_{2}=0.1, c_{3}=0.9, c_{4}=0.6, s_{1}=0.6, s_{2}=0.1, b=1.1, d_{1}=0.9, d_{2}=0.1, d_{3}=0.1, \alpha=0.98$, and $\tau=13.5$; the initial values are $r(t)=0.1, p(t)=1, u(t)=2(t \in[-\tau, 0])$

$(0.3571,0,1.875)$. Because $b / d_{3}=3, \theta_{1}=1.875$, and $\theta_{2}=10$, the inequality $\theta_{2}>b / d_{3}>\theta_{1}$ holds. By Theorem 1 the equilibrium $(0,0,3)$ is unstable. From (15) and (16) we have $a_{1} \approx 0.0197, a_{2} \approx-0.2862, a_{3} \approx 0.0038, a_{4} \approx 0.0179$. Equation (11) has a positive real root $v_{a} \approx 0.2677$. From (13) we obtain $M_{a} \approx 0.7443$ and $N_{a} \approx 7.8279$. Because the discriminant $\Delta_{a} \approx 0.000866>0$ and $a_{2}<0$, the equation $f_{a}(\xi)=0$ has two unequal positive real roots by Lemma 2 , and the larger root $\xi_{+} \approx 0.3819$. So equation (20) has a positive real $\operatorname{root} \xi_{a}$ satisfying $\xi_{a}^{\alpha}=\xi_{+}$. Substituting $\xi_{a}$ into (18), we get $\cos \tau \xi_{a} \approx 0.3677>0$. So we obtain the Hopf bifurcation critical value $\tau_{a} \approx 13.5888$ by using (21). Therefore, by Theorem 2 the equilibrium $(0.3571,0,1.875)$ is asymptotically stable when $\tau \in[0,13.5888)$ as illustrated in Fig. 2 (where $\tau=13.5$ ); Otherwise, the equilibrium $(0.3571,0,1.875)$ is unstable, and a Hopf bifurcation emerges at $\tau \approx 13.5888$ (see Fig. 3, where $\tau=13.59$ ).

\section{Conclusions}

We have proposed a delayed fractional-order differential equation model that reflects the interaction among rice, weeds, and inorganic fertilizer in a paddy ecosystem. If $\alpha=1$ and $c_{3}=c_{4}=1$, then system (5) degenerates into system (4), which was studied in [12]. The equilibria and their existence conditions of system (5) are the same as those of system (4), where those conditions are related to the relative mortality of rice and weeds, $\theta_{1}$ and $\theta_{2}$, and to the ratio of fertilizer supply and loss $b / d_{3}$, but not to other parameters. Under the condition $b / d_{3}<\min \left\{\theta_{1}, \theta_{2}\right\}$, there is a unique stable equilibrium $\left(0,0, u_{1}^{*}\right)$ in each of the two systems. If $b / d_{3}>\max \left\{\theta_{1}, \theta_{2}\right\}$, then each of the two systems has three equilibria: the equilibrium for rice and weeds extinction $\left(0,0, u_{1}^{*}\right)$, the equilibrium for weeds extinction $\left(r_{2}^{*}, 0, u_{2}^{*}\right)$, and the equilibrium for rice extinction $\left(0, p_{3}^{*}, u_{3}^{*}\right)$, where the equilibrium $\left(0,0, u_{1}^{*}\right)$ is unstable, and $\left(r_{2}^{*}, 0, u_{2}^{*}\right)$ is also unstable when $\theta_{1}>\theta_{2}$, or $\left(0, p_{3}^{*}, u_{3}^{*}\right)$ is unstable when $\theta_{1}<\theta_{2}$. Under the condition $\theta_{1}<b / d_{3}<\theta_{2}$, there exist two equilibria $\left(0,0, u_{1}^{*}\right)$ and $\left(r_{2}^{*}, 0, u_{2}^{*}\right)$. Under the condition $\theta_{2}<b / d_{3}<\theta_{1}$, there exist two equilibria $\left(0,0, u_{1}^{*}\right)$ and $\left(0, p_{3}^{*}, u_{3}^{*}\right)$. 


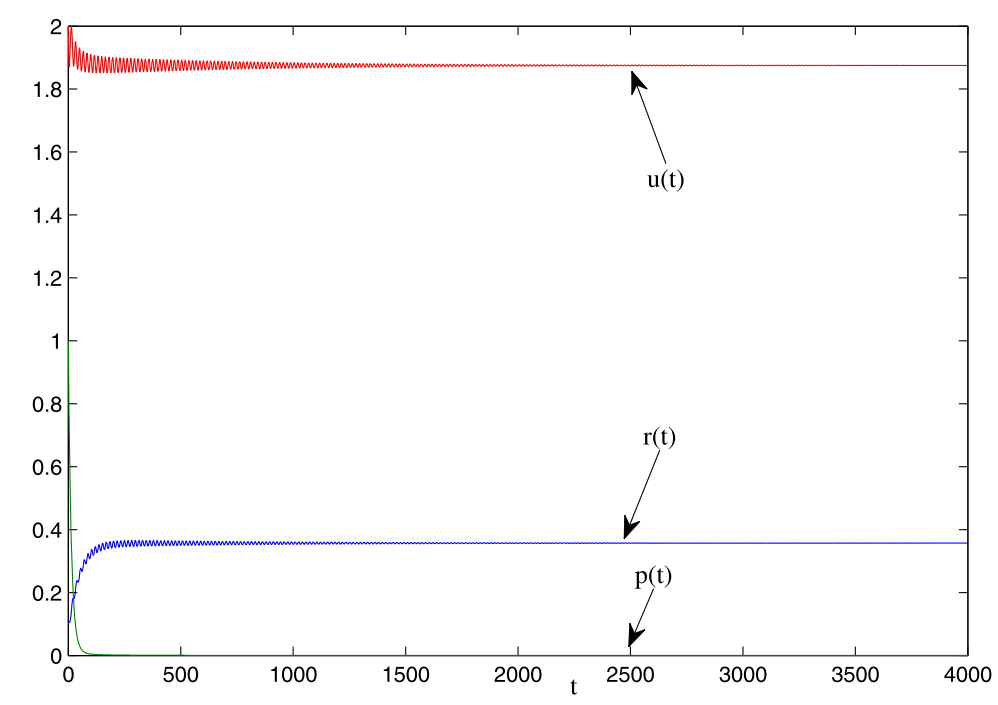

Figure 2 The asymptotical stability with delay $\tau=13.5$. The parameters of system (5) are $c_{1}=0.8, c_{2}=0.1$, $c_{3}=0.9, c_{4}=0.6, s_{1}=0.6, s_{2}=0.1, b=0.3, d_{1}=0.9, d_{2}=0.1, d_{3}=0.1$, and $\alpha=0.98$; the initial values are $r(t)=0.1, p(t)=1, u(t)=2(t \in[-\tau, 0])$. The Hopf bifurcation critical value $\tau_{a} \approx 13.5888$. It depicts the asymptotical stability of the equilibrium $\left(r_{2}^{*}, p_{2}^{*}, u_{2}^{*}\right) \approx(0.3571,0,1.875)$ with time delay $\tau=13.5<\tau_{a}$

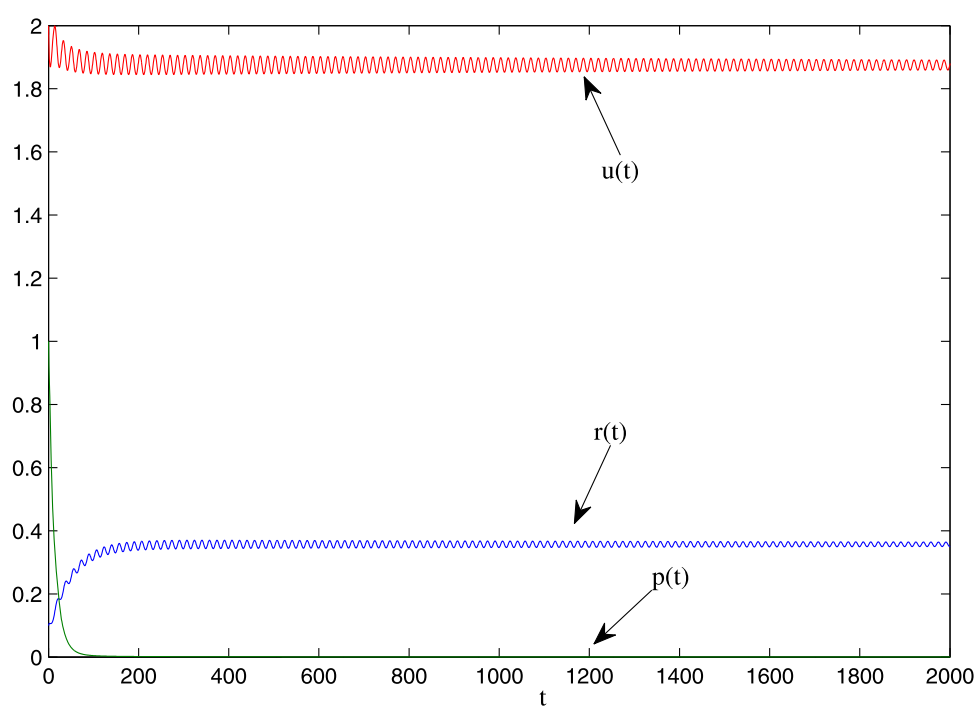

Figure 3 A periodic oscillation with delay $\tau=13.59$. The parameters of system (5) are $c_{1}=0.8, c_{2}=0.1$, $c_{3}=0.9, c_{4}=0.6, s_{1}=0.6, s_{2}=0.1, b=0.3, d_{1}=0.9, d_{2}=0.1, d_{3}=0.1$, and $\alpha=0.98$; the initial values are $r(t)=0.1, p(t)=1, u(t)=2(t \in[-\tau, 0])$. The Hopf bifurcation critical value $\tau_{a} \approx 13.5888$. It depicts a periodic oscillation bifurcating from the equilibrium $\left(r_{2}^{*}, p_{2}^{*}, u_{2}^{*}\right) \approx(0.3571,0,1.875)$ with time delay $\tau=13.59>\tau_{a}$

We also generalize the conditions of stabilities of equilibria and Hopf bifurcation obtained by Wang et al. [12]. If we take $\alpha=1$ and $c_{3}=c_{4}=1$, then we have $a_{1}=a_{3}=0$, $a_{2}=\left(s_{1} r_{2}^{*}+d_{3}\right)^{2}-2 s_{1} r_{2}^{*} d_{1}$, and $a_{4}=s_{1}^{2} r_{2}^{* 2} d_{1}^{2}\left(1-c_{1}^{2}\right)$ from (15) and (16). Equation (11) has a positive root $v_{a}=2 \sqrt{a_{4}}$. So we obtain $\Delta_{a}=-a_{2}-2 \sqrt{a_{4}}$ from (14). If $\Delta_{a}<0$, then we have

$$
\left(s_{1} r_{2}^{*}+d_{3}\right)^{2}>2 s_{1} r_{2}^{*} d_{1}\left(1-\sqrt{1-c_{1}^{2}}\right) .
$$


It is condition (5) of Theorem 2 in [12]. Similarly, from $\Delta_{a}>0$ we can obtain condition (6) in [12]. Moreover, substituting $\alpha=1$ and $c_{3}=1$ into the Hopf bifurcation critical value formulas (21) and (22), we can obtain formulas (12) and (13) in [12], respectively.

Funding

This work was partly supported by Hunan province science and technology project (grants 2015JC3101), and the scientific research fund of Hunan provincial education department (grants 14B090).

\section{Competing interests}

The authors declare that they have no competing interests.

\section{Authors' contributions}

All authors contributed equally to the writing of this paper. All authors read and approved the final version of the manuscript.

\section{Publisher's Note}

Springer Nature remains neutral with regard to jurisdictional claims in published maps and institutional affiliations.

\section{Received: 3 March 2018 Accepted: 15 July 2018 Published online: 10 September 2018}

\section{References}

1. Abbas, S., Banerjee, M., Hungerbühler, N.: Existence, uniqueness and stability analysis of allelopathic stimulatory phytoplankton model. J. Math. Anal. Appl. 367(1), 249-259 (2010)

2. Abbas, S., Sen, M., Banerjee, M.: Almost periodic solution of a non-autonomous model of phytoplankton allelopathy Nonlinear Dyn. 67, 203-214 (2012)

3. Chen, C.: Marine ecosystem dynamics and model Higher Education Press, Beijing (2003) (in Chinese)

4. Dai, C., Zhao, M., Yu, H.: Dynamics induced by delay in a nutrient-phytoplankton model with diffusion. Ecol. Complex. 26, 29-36 (2016)

5. Hofmann, E.E., Amblerl, J.W.: Plankton dynamics on the outer southeastern U.S. continental shelf. Part II: a time-dependent biological model. J. Mar. Res. 46, 883-917 (1988)

6. Jang, S.R.-J., Allen, E.J.: Deterministic and stochastic nutrient-phytoplankton-zooplankton models with periodic toxin producing phytoplankton. Appl. Math. Comput. 271, 52-67 (2015)

7. Yadigar, S., Sergei, P.: Mathematical modelling of plankton-oxygen dynamics under the climate change. Bull. Math. Biol. 77, 2325-2353 (2015)

8. Sharma, A., Sharma, A.K., Agnihotri, K.: Analysis of a toxin producing phytoplankton-zooplankton interaction with Holling IV type scheme and time delay. Nonlinear Dyn. 81(1-2), 13-25 (2015)

9. Zhang, T., Wang, W.: Hopf bifurcation and bistability of a nutrient-phytoplankton-zooplankton model. Appl. Math. Model. 36, 6225-6235 (2012)

10. Zhao, H., Huang, X., Zhang, X.: Hopf bifurcation and harvesting control of a bioeconomic plankton model with delay and diffusion terms. Phys. A, Stat. Mech. Appl. 421, 300-315 (2015)

11. Zhao, J., Tian, J.P., Wei, J.: Minimal model of plankton systems revisited with spatial diffusion and maturation delay. Bull. Math. Biol. 78, 381-412 (2016)

12. Wang, Y., Zhou, X., Wu, Z., Zhou, T.: Stability of a paddy ecosystem with time delay. In: International Conference on Applied Mathematics, Modelling and Statistics Application, vol. 1, pp. 1-5 (2017)

13. Xiang, M., Wu, Z., Zhou, T.: Analysis of the interaction among weed, inorganic fertilizer and herbivore in paddy ecosystem in fallow season. Int. J. Biomath. 10(8), Article ID 1750120 (2017). https://doi.org/10.1142/S1793524517501200

14. Xiang, M., Wu, Z., Zhou, T.: Stability of a paddy ecosystem in fallow season. J. Biomath. 32(1), 49-56 (2017) (in Chinese)

15. Ahmed, E., El-Sayed, A.M.A., El-Saka, H.A.A.: Equilibrium points, stability and numerical solutions of fractional-order predator-prey and rabies models. J. Math. Anal. Appl. 325(1), 542-553 (2007). https://doi.org/10.1016/j.jmaa.2006.01.087

16. Das, S., Gupta, P.K.: A mathematical model on fractional Lotka-Volterra equations. J. Theor. Biol. 277(1), 1-6 (2011). https://doi.org/10.1016/j.jtbi.2011.01.034

17. Ghaziani, R.K., Alidousti, J., Eshkaftaki, A.B.: Stability and dynamics of a fractional order Leslie-Gower prey-predator model. Appl. Math. Model. 40(3), 2075-2086 (2016). https://doi.org/10.1016/j.apm.2015.09.014

18. Javidi, M., Ahmad, B.: Dynamic analysis of time fractional order phytoplankton-toxic phytoplankton-zooplankton system. Ecol. Model. 318(Supplement C), 8-18 (2015). https://doi.org/10.1016/j.ecolmodel.2015.06.016

19. Javidi, M., Nyamoradi, N.: Dynamic analysis of a fractional order prey-predator interaction with harvesting. Appl. Math. Model. 37(20), 8946-8956 (2013). https://doi.org/10.1016/j.apm.2013.04.024

20. Nosrati, K., Shafiee, M.: Dynamic analysis of fractional-order singular Holling type-ll predator-prey system. Appl. Math. Comput. 313, 159-179 (2017)

21. Abdelouahab, M.S., Hamri, N.E., Wang, J.W.: Hopf bifurcation and chaos in fractional-order modified hybrid optical system. Nonlinear Dyn. 69(1-2), 275-284 (2012)

22. Latha, V.P., Rihan, F.A., Rakkiyappan, R., Velmurugan, G.: A fractional-order delay differential model for Ebola infection and CD8+ T-cells response: stability analysis and Hopf bifurcation. Int. J. Biomath. 10, Article ID 1750111 (2017)

23. Li, X., Wu, R.: Hopf bifurcation analysis of a new commensurate fractional-order hyperchaotic system. Nonlinear Dyn. 78(1), 279-288 (2014)

24. Sun, Q., Xiao, M., Tao, B., Jiang, G., Cao, J., Zhang, F., Huang, C.: Hopf bifurcation analysis in a fractional-order survival red blood cells model and $P D^{\alpha}$ control. Adv. Differ. Equ. 2018(1), Article ID 10 (2018) 
25. Tao, B., Xiao, M., Sun, Q., Cao, J.: Hopf bifurcation analysis of a delayed fractional-order genetic regulatory network model. Neurocomputing 275, 677-686 (2018). https://doi.org/10.1016/j.neucom.2017.09.018

26. Xiao, M., Jiang, G., Cao, J., Zheng, W.: Local bifurcation analysis of a delayed fractional-order dynamic model of dual congestion control algorithms. IEEE/CAA J. Autom. Sin. 4(2), 1-9 (2017)

27. Xiao, M., Zheng, W.X., Lin, J., Jiang, G., Zhao, L., Cao, J.: Fractional-order PD control at Hopf bifurcations in delayed fractional-order small-world networks. J. Franklin Inst. 354(17), 7643-7667 (2017)

28. Wang, H., Yu, Y., Wen, G., Zhang, S.: Stability analysis of fractional-order neural networks with time delay. Neural Process. Lett. 42(2), 479-500 (2015)

29. Bhalekar, S., Daftardar-Gejji, V:: A predictor-corrector scheme for solving nonlinear delay differential equations of fractional order. J. Fract. Calc. Appl. 1(5), 1-9 (2011)

Submit your manuscript to a SpringerOpen ${ }^{\circ}$ journal and benefit from:

- Convenient online submission

- Rigorous peer review

- Open access: articles freely available online

- High visibility within the field

- Retaining the copyright to your article

Submit your next manuscript at $\gg$ springeropen.com 